\title{
A NEW ZZ CETI WHITE DWARF PULSATOR: G30-20
}

\author{
Anjum S. Mukadam, ${ }^{1,2}$ S. O. Kepler, ${ }^{3}$ D. E. Winget, ${ }^{1,2}$ and P. Bergeron ${ }^{4}$ \\ Received 2002 February 25; accepted 2002 July 23
}

\begin{abstract}
We report the discovery of a new hydrogen atmosphere variable white dwarf (DAV; ZZ Ceti), G30-20, a high-amplitude, long-period pulsator. The Fourier transform of its light curve shows power around 1050 $1125 \mathrm{~s}$. We suspect the existence of multiple periodicities in this region, probably corresponding to different radial order $(k)$ modes, but our observations are not long enough to resolve them. This discovery increases the number of known DAVs to 32 . Our best fit to its optical spectrum gives $T_{\text {eff }}=11,070 \pm 180 \mathrm{~K}$ and $\log g=7.95 \pm 0.06$. This implies that G30-20 is the coolest known DAV.

Subject headings: stars: individual (G30-20, WD 2347+128) — stars: oscillations — stars: variables: other — white dwarfs
\end{abstract}

\section{INTRODUCTION}

White dwarfs are the end points of most stars in the universe, and their structure provides constraints on their prior evolution. Their high gravity and temperature make them good cosmic laboratories to study physics under extreme conditions. As white dwarfs cool, they pass through an instability strip in which we observe them as nonradial $g$ mode pulsators. Pulsating white dwarfs offer us a chance to study their internal structure through the techniques of asteroseismology.

As $80 \%$ of all white dwarf stars have atmospheres dominated by hydrogen (DAs), to understand the pulsating DAs (DAVs) is to understand the most common type of white dwarf. DAVs are not defective or special in any way. All DAs have been shown to pulsate when their temperatures reach the ZZ Ceti instability strip (Robinson 1979; Fontaine et al. 1985), i.e., pulsation is an evolutionary phase.

The DAV strip is empirically found between 11,200 and $12,500 \mathrm{~K}, \log g \approx 8$, using model atmospheres (Bergeron et al. 1995b) that treat convection with ML $2 / \alpha=0.6(\operatorname{see} \S 5)$. The DAV white dwarfs are also known as ZZ Ceti stars after ZZ Ceti, the prototype of the class. Their observed periods typically range from 100 to $1000 \mathrm{~s}$, which is consistent with nonradial $g$-mode pulsations (see the review paper Winget 1998 and references therein). As each mode we observe gives us information on a different part of the star, the more modes we detect, the better our understanding of the stellar structure. Clemens (1993) was the first to combine data from different hot DAVs (HDAVs) to paint a coherent picture of them as a group. Kleinman et al. (1998) observed G29-38, a cool DAV (CDAV), over many observing seasons, combining modes that come and go with time to provide the largest coherent set of modes seen in any single DAV. We currently know 31 DAVs; additional pulsators and additional modes in known pulsators will help us understand the DAVs as a class.

\footnotetext{
${ }^{1}$ University of Texas at Austin, 17.310 RLM Hall, Austin, TX 78712; anjum@astro.as.utexas.edu,dew@bullwinkle.as.utexas.edu.

${ }^{2}$ McDonald Observatory, Fort Davis, TX 79734.

${ }^{3}$ Instituto de Física, Universidade Federal do Rio Grande do Sul, 91501 970 Porto Alegre, RS Brazil; kepler@if.ufrgs.br.

${ }^{4}$ Département de Physique, Université de Montréal, CP 6128, Succ. Centre-Ville, Montréal, QC, H3C 3J7, Canada; bergeron@ ASTRO.UMontreal.CA.
}

Pulsation models indicate that the limits of the ZZ Ceti instability strip depend on the effective temperature and mass of the star (Bradley \& Winget 1994). The blue edge of the strip is expected to vary from $13,460 \mathrm{~K}$ for a $0.8 M_{\odot}$ DA to $12,000 \mathrm{~K}$ for a $0.4 M_{\odot}$ DA star for an interior ML 3 convection prescription (Kepler \& Bradley 1995). Most model atmospheres of DAV stars treat convection with a mixing length prescription assuming some parameterization, the choice of which can shift the edges of the instability strip in temperature by a few thousand K (Bergeron et al. 1995b; Koester \& Allard 2000). Pulsation models predict a red edge at temperatures as low as $8000-10,000 \mathrm{~K}$ (Winget 1981; Winget et al. 1982; Dolez \& Vauclair 1981; Hansen, Winget, \& Kawaler 1985).

As models cool below the blue edge, the partial ionization zone moves deeper into the star, and driving is provided for modes of lower and lower frequency. The models stop showing excited modes with periods shorter than or equal to $1500 \mathrm{~s}$ at temperatures of $8000 \mathrm{~K}$. Longer period modes could still exist at even lower temperatures. The surface atmospheric layers may not reflect internal waves well at long timescales, and the energy of an excited mode may leak through the surface, eventually damping it (Hansen et al. 1985). Brickhill (1983) showed that for a given model, the restricted frequency interval where pulsations of large amplitude are observable moves into a frequency range smaller than the critical value for all $g$-modes, as the effective temperature of the models is decreased. At this critical value, the surface flux amplitudes of pulsation modes decrease as the delay associated with the convective zone increases as a result of turbulent viscosity, and large changes in radial and pressure perturbations are produced in models. Brickhill (1983) found the red edge at models with $T_{\text {eff }}=11,000 \mathrm{~K}$ and $\log g=8$. Bradley \& Winget (1994) were unable to find a red edge with their models. Wu \& Goldreich (1999) get a maximum period of about $2300 \mathrm{~s}$ from nonadiabatic calculations of overstable modes. Including convective overshoot, they get a maximum period of $1400 \mathrm{~s}$ for overstable modes, which is comparable to the observed maximum period of $1200 \mathrm{~s}$ for detected modes in CDAVs. Determining the location of the red edge is made difficult by convective overshoot and nonlinear effects.

Observable amplitudes of detected modes in CDAVs decline for periods in excess of $1000 \mathrm{~s}$ (see, e.g., Fig. 5 from Clemens 1995). This suggests that the observational red 
edge at a maximum period of $1200 \mathrm{~s}$ is genuine. Kanaan et al. (2000) and Kanaan, Kepler, \& Winget (2002) conclude that the observed red edge for the ZZ Ceti instability strip at $11,000 \mathrm{~K}$ is not an observational selection effect, as the upper limit set by noise is a factor of 50 smaller than typical pulsation amplitudes. The blue edge of the instability strip is currently defined by GD 165 (Koester \& Allard 2000) or G226-29 (Bergeron et al. 1995b). The red edge of the strip is observationally defined by three ZZ Ceti stars R808, GD 154, and G38-29 (Bergeron et al. 1995b; Koester \& Allard 2000). Wu \& Goldreich $(1999,2001)$ suspect that convective driving allows velocity variations to become more observable than luminosity variations toward the red edge. Kotak et al. (2002) got time-resolved spectra of three nonpulsating DA white dwarfs with temperatures slightly cooler than the ZZ Ceti strip, but did not detect any significant periodic velocity variations. Finding more DAVs at different temperatures and masses will improve our observational determination of the edges of the ZZ Ceti strip, as well as determine its mass dependence (Kepler et al. 2000).

The HDAV stars are found to exhibit extreme amplitude and frequency stability, making them reliable clocks. When such stable clocks have an orbiting planet around them, their reflex motion around the center of mass of the system becomes measurable, providing a means to detect the planet (Kepler et al. 1990, 1991; Mukadam, Winget, \& Kepler 2001). The success of a planet search project based on this technique rests on finding a statistically significant number of HDAV stars. We discovered G30-20 in our search for additional HDAV stars.

\section{G30-20: A HIGH-PRIORITY ZZ CETI CANDIDATE}

Greenstein \& Liebert (1990) published high signal-tonoise spectrophotometric data for G30-20 (WD 2347+128; EGGR 405; $B=15.9$ ). Dolez, Vauclair, \& Koester (1991) fitted this data to a grid of pure hydrogen model atmospheres with local thermal equilibrium (LTE) and found a temperature of $T_{\text {eff }}=11,000 \pm 100 \mathrm{~K}$ with $\log g=8.50 \pm$ 0.10 for $\mathrm{G} 30-20$.

Greenstein (1982) found that 14 DAs lie in the strip, $-0.41 \geq G-R \geq-0.29$, on the AB 79 scale (Oke \& Gunn $1983)$ and concluded that $(G-R)$ color proves to be an excellent temperature indicator for DAs. From the magnitudes given by Greenstein \& Liebert (1990), we compute the following color indices: $V-I=-0.415, \quad G-R=-0.28$, $U-V=0.43, U-G=0.49$, and $B-V=-0.05$. G30-20 with $G-R=-0.28$ is close to the red edge of the instability

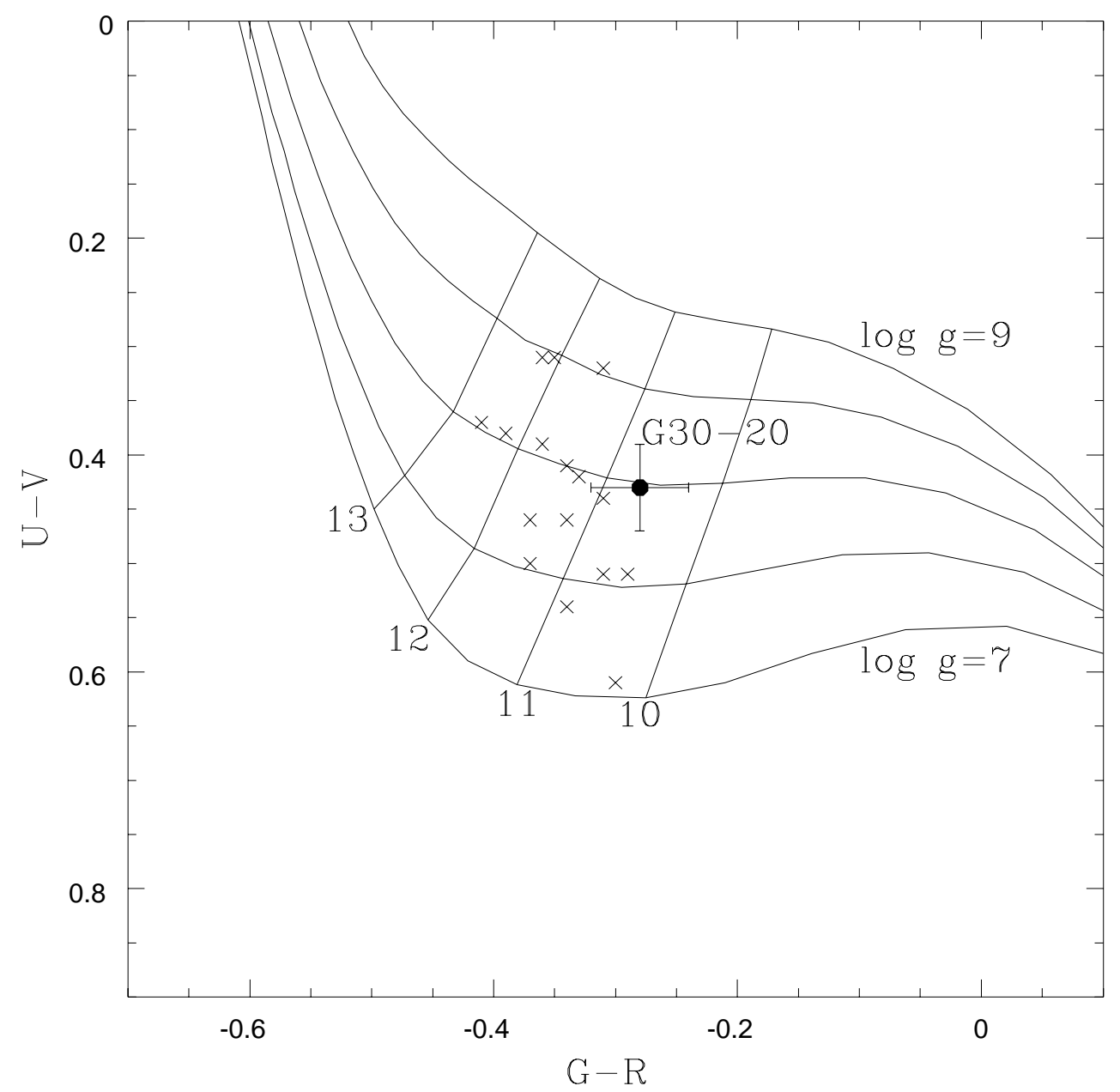

FIG. 1.-Pure hydrogen DA models by Bergeron et al. (2001) plotted as a function of the color indices $G-R$ and $U-V$, with $T_{\text {eff }}$ indicated in units of $10^{3} \mathrm{~K}$ and $\log g$ values of 7.0 (bottom) to 9.0 (top). For G30-20, we obtained $G-R=-0.28, U-V=0.43$ from high signal-to-noise spectrophotometric data (Greenstein \& Liebert 1990), with an assumed uncertainty of 0.04 mag. The location of G30-20 in the ZZ Ceti instability strip is shown, along with the error bars. We have plotted the known ZZ Ceti stars from Greenstein (1984) as crosses to show the mass dependence of the instability strip. 
TABLE 1

Journal of Observations for Data Acquired on G30-20 in 2001 December

\begin{tabular}{ccccc}
\hline \hline Observing Run & $\begin{array}{c}\text { Date } \\
\text { (UTC) }\end{array}$ & $\begin{array}{c}\text { Time } \\
\text { (UTC) }\end{array}$ & $\begin{array}{c}\text { Duration } \\
\text { (hr) }\end{array}$ & $\begin{array}{c}\text { Integration Time } \\
\text { (s) }\end{array}$ \\
\hline asm-0094............ & $2001 \operatorname{Dec} 19$ & $01: 15: 00$ & 2.21 & 10 \\
asm-0099........... & 2001 Dec 20 & $01: 03: 10$ & 2.95 & 5 \\
\hline
\end{tabular}

NotE.-Data acquired at the $2.1 \mathrm{~m}$ telescope of McDonald Observatory with a three-star photometer.

strip. Colors for pure hydrogen DA model atmospheres published by Bergeron, Wesemael, \& Beauchamp (1995a) and Bergeron, Leggett, \& Ruiz (2001) allow us to estimate the temperature of $\mathrm{G} 30-20$ to be $10,700 \pm 300 \mathrm{~K}$ with $\log g=8.0 \pm 0.3$, as indicated in Figure 1 .

\section{OBSERVATIONS}

We obtained time-series aperture photometry on G30-20 in 2001 December at the $2.1 \mathrm{~m}$ telescope at McDonald Observatory. The data were acquired using " P3Mudgee," a three-star photometer (Kleinman, Nather, \& Phillips 1996). We observed unfiltered light to maximize the signal-to-noise ratio; $g$-mode pulsations in white dwarfs do not change phase with wavelength (Robinson, Kepler, \& Nather 1982). We present the journal of observations in Table 1 .

Dolez et al. (1991) state that G30-20 is a nonvariable, setting amplitude detection limits at a few mmag. ${ }^{5}$ Our light curve of the star clearly shows beating effects; beating can destroy pulsations in the light curve for up to a few hours, depending on the amplitude of the pulsation and the magnitude of the star, both of which dictate the signal-to-noise ratio. It is possible to obtain an essentially constant light curve of G30-20 for up to an hour, depending on the time of observation. Hence, our observations are not inconsistent with their observations.

51 mmag equals $0.1086 \%$ change in intensity.
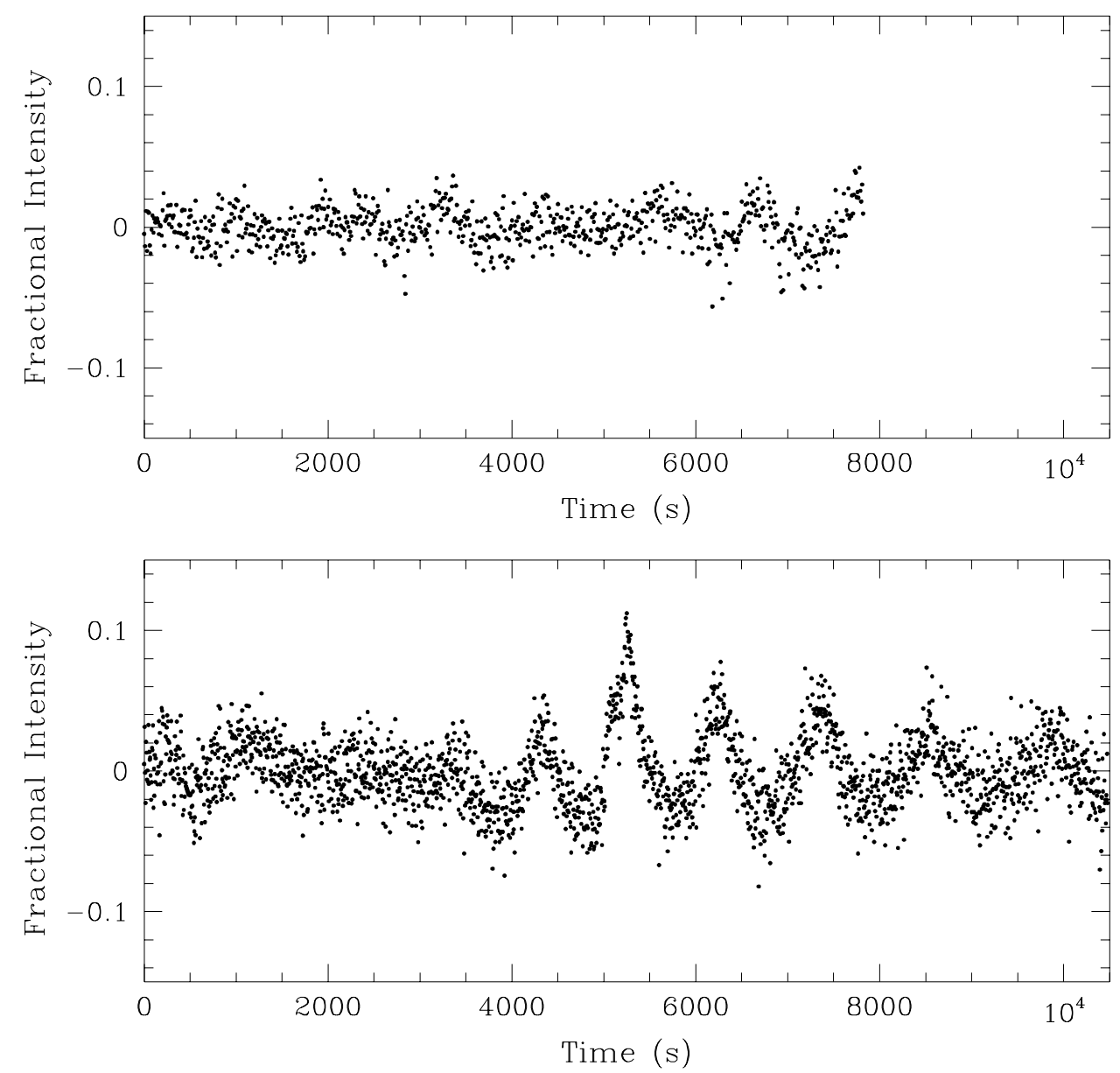

FIG. 2.- Reduced light curves of G30-20 obtained on 2001 December 19 and 20, respectively, at the $2.1 \mathrm{~m}$ telescope of McDonald Observatory, using a three-star photometer. The top panel shows a $2.21 \mathrm{hr}$ long light curve with an integration time of $10 \mathrm{~s}$. The bottom panel shows a $2.95 \mathrm{hr}$ long light curve with an integration time of $5 \mathrm{~s}$. 


\section{DATA REDUCTION}

We reduced and analyzed the data in a manner described by Nather et al. (1990) and Kepler (1993), correcting for extinction and sky variations. After this preliminary reduction, we brought the data to the same fractional amplitude scale and converted the times of observations to barycentric coordinated time TCB (Standish 1998). The light curves of the two runs reduced in this manner are shown in Figure 2. We computed a discrete Fourier transform (DFT) for each individual run and the combined data. These are shown in Figure 3, along with the corresponding "window" pattern, which is what a single frequency in that data set should look like.

We conclude that this ZZ Ceti star is a high-amplitude long-period variable. Cool ZZ Ceti stars show high amplitudes in the range of $10-50 \mathrm{mma}$ (milli-modulation amplitude), where $1 \mathrm{mma}$ equals $0.1 \%$ change in intensity. For example, O’Donoghue, Warner, \& Cropper (1992) detected an amplitude of $56 \mathrm{mma}$ for the $617.87 \mathrm{~s}$ period in BPM 31594, while Dolez (1998) detected a period at $541 \mathrm{~s}$ in HL Tau 76 with an amplitude of 37.3 mmag. Our observations are not long enough to resolve the power evident in the region $1050-1125 \mathrm{~s}$. We suspect the existence of multiple periodicities in this region that might correspond to different $k$ modes. The combined DFT from both data sets shows a peak at $1068 \mathrm{~s}$, with an amplitude of $13.8 \mathrm{mma}$. It will be necessary to observe G30-20 long enough to resolve its pulsation spectrum in order to be able to use the pulsations to study its structure.

\section{COOLEST KNOWN DAV YET}

Bergeron et al. (1995b) considered various parameterizations of the mixing-length theory (MLT) to treat convection in models of ZZ Ceti stars. Böhm \& Cassinelli (1971) describe the ML 2 version of convection, assuming the ratio of the mixing length $(l)$ to the pressure scale height $(H)$, $\alpha \equiv l / H=1$. Bergeron et al. (1995b) analyzed optical and ultraviolet (UV) spectrophotometric data of ZZ Ceti stars and found that model atmospheres calculated using the ML 2 version, assuming $\alpha=0.6$, provide an internal consistency between the optical and UV temperature estimates, the observed photometry, the trigonometric parallax measurements, and the gravitational redshift masses. We obtained a best value of $T_{\text {eff }}=11,070 \pm 180 \mathrm{~K}$ and $\log g=7.95 \pm 0.06$ by fitting Balmer line profiles from a high signal-to-noise flux-calibrated optical spectrum of G30-20, obtained by C. K. J. Moran, with these model atmospheres. The model fits are shown in Figure 4. Bergeron et al. (1995b) have argued that the Balmer line profiles from $\mathrm{H} \beta$ to $\mathrm{H} 9$ are the best estimators of temperature and surface gravity.
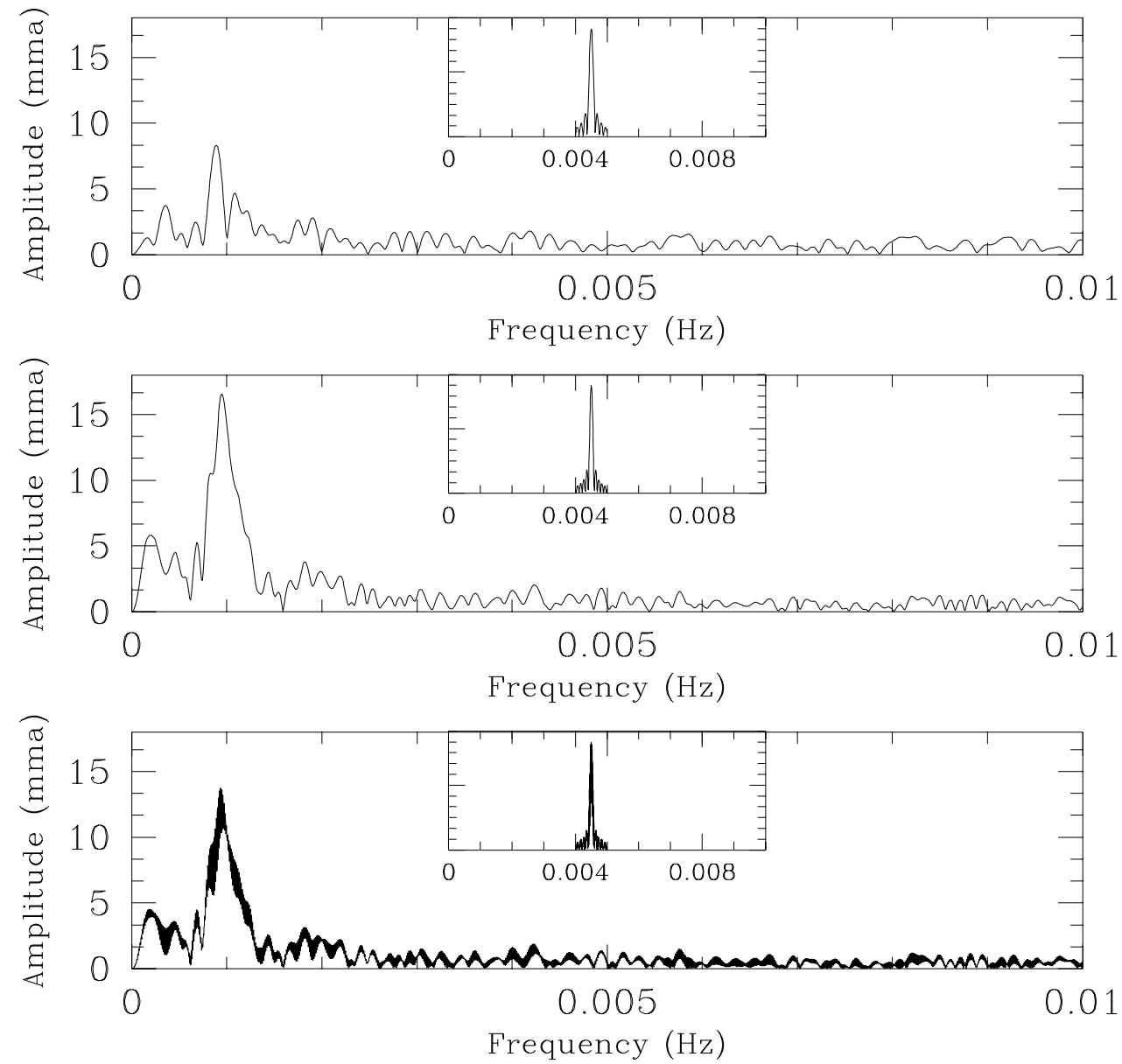

FIG. 3.-Top two panels show the discrete Fourier transform (DFT) of G30-20 from the individual runs on 2001 December 19 and 20 , respectively. The bot-

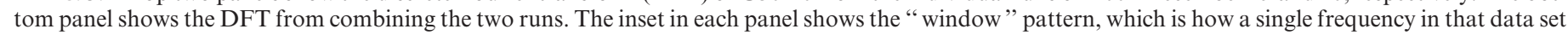
should look. 
The formal $1 \sigma$ internal errors of the fitting parameters are $60 \mathrm{~K}$ in $T_{\text {eff }}$ and 0.04 in $\log g$. Such small internal errors only represent the ability and the sensitivity of the model spectra to match the observed spectra (Bergeron et al. 1995b). The error budget is actually dominated by external errors, which originate mostly from uncertainties of the flux calibration. We have estimated this external error by fitting the four ZZ Ceti stars (G38-29, GD 66, G238-53, and G2938 ) found in common between the sample from which the spectrum of G30-20 was drawn and that of Bergeron et al. (1995b). The standard deviations between these independent samples are $180 \mathrm{~K}$ in $T_{\text {eff }}$ and 0.06 in $\log g$, which we adopt here as our formal errors of the fit to G30-20.

G30-20 is currently the coolest known DAV at $T_{\text {eff }}=11,070 \mathrm{~K}$ with $\log g=7.95$ among the ZZ Ceti stars that do have a published temperature determination (Bradley 2000; Bergeron et al. 1995b). ${ }^{6}$ Using theoretical evolutionary models (Wood 1995) with thick hydrogen layers, we derive a mass of $M_{*}=0.58 M_{\odot}$, an absolute visual magnitude of $M_{v}=11.8$, and a distance of $d=70 \mathrm{pc}$ from Earth. G30-20 is thus close to the observational red edge of the ZZ Ceti instability strip.

We acknowledge and thank C. K. J. Moran for taking high signal-to-noise spectroscopic data that led to a better determination of the temperature of this star, quoted in this paper. We also acknowledge the NSF grant AST-9876730, NASA grant NAG 5-9321, and STSI GO-08254 for their funding and support. We thank the careful referee for helping us improve the paper.

${ }^{6}$ The ZZ Ceti stars KUV 08368+4026, KUV 11370+4222, EC 14012-1446, and EC 23487-2424 do not yet have published temperature determinations (Bradley 2000).

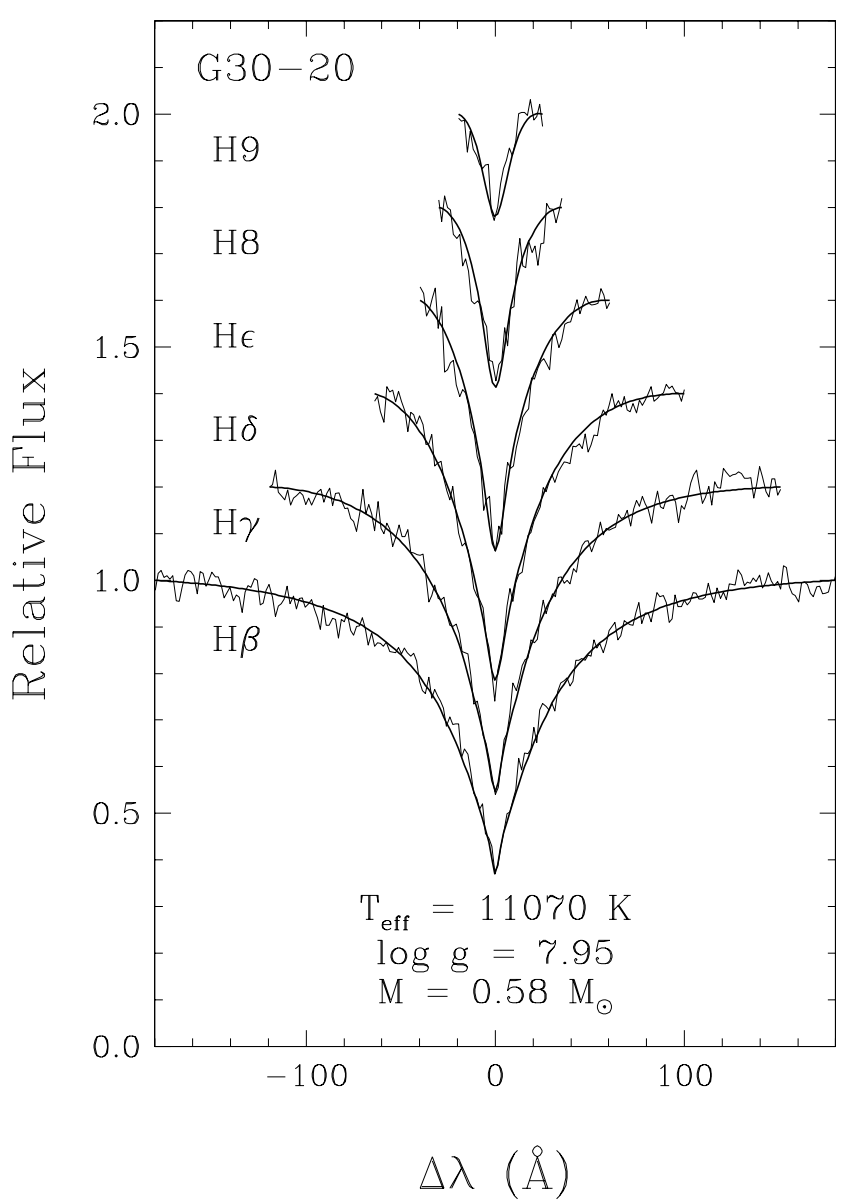

FIG. 4.- - High signal-to-noise optical spectrum of G30-20, obtained by C. K. J. Moran, yielded a best fit of $T_{\text {eff }}=11,070 \pm 180 \mathrm{~K}$ with $\log g=7.95 \pm 0.06$, using the ML 2 model atmospheres with $\alpha=0.6$.

\section{REFERENCES}

Bergeron, P., Leggett, S. K., \& Ruiz, M. 2001, ApJS, 133, 413

Bergeron, P., Wesemael, F., \& Beauchamp, A. 1995a, PASP, 107, 1047

Bergeron, P., Wesemael, F., Lamontagne, R., Fontaine, G., Saffer, R. A., \& Allard, N. F. 1995b, ApJ, 449, 258

Böhm, K. H., \& Cassinelli, J. 1971, A\&A, 12, 21

Bradley, P. A. 2000, Baltic Astron., 9, 485

Bradley, P. A., \& Winget, D. E. 1994, ApJ, 421, 236

Brickhill, A. J. 1983, MNRAS, 204, 537

Clemens, J. C. 1993, Ph.D. thesis, Univ. Texas, Austin . 1995, Baltic Astron., 4, 142

Dolez, N. 1998, Baltic Astron., 7, 153

Dolez, N., \& Vauclair, G. 1981, A\&A, 102, 375

Dolez, N., Vauclair, G., \& Koester, D. 1991, in Proc. 7th European Workshop, White Dwarfs, ed. G. Vauclair \& E. Sion (Dordrecht: Kluwer), 361

Fontaine, G., Bergeron, P., Lacombe, P., Lamontagne, R., \& Talon, A. 1985, AJ, 90, 1094

Greenstein, J. L. 1982, ApJ, 258, 661

. 1984, ApJ, 276, 602

Greenstein, J. L., \& Liebert, J. W. 1990, ApJ, 360, 662

Hansen, C. J., Winget, D. E., \& Kawaler, S. D. 1985, ApJ, 297, 544

Kanaan, A., Kepler, S. O., \& Winget, D. E. 2002, A\&A, 389, 896

Kanaan, A., Winget, D. E., Kepler, S. O., \& Montgomery, M. H. 2000, in ASP Conf. Ser. 203, The Impact of Large-Scale Surveys on Pulsating Star Research, ed. L. Szabados \& D. W. Kurtz (San Francisco: ASP), 518

Kepler, S. O. 1993, Baltic Astron., 2, 515

Kepler, S. O., \& Bradley, P. A. 1995, Baltic Astron., 4, 166

Kepler, S. O., da Costa, A. F. M., Giovannini, O., \& Koester, D. 2000, Baltic Astron., 9, 125
Kepler, S. O., et al. 1990, ApJ, 357, 204 . 1991, ApJ, 378, L45

Kleinman, S. J., Nather, R. E., \& Phillips, T. 1996, PASP, 108, 356

Kleinman, S. J., et al. 1998, ApJ, 495, 424

Koester, D., \& Allard, N. F. 2000, Baltic Astron., 9, 119

Kotak, R., et al. 2002, A\&A, 391, 1005

Mukadam, A. S., Winget, D. E., \& Kepler, S. O. 2001, in ASP Conf. Ser. 226, 12th European Workshop on White Dwarfs, ed. L. Provencal, H. L. Shipman, J. MacDonald, \& S. Goodchild (San Francisco: ASP), 337

Nather, R. E., Winget, D. E., Clemens, J. C., Hansen, C. J., \& Hine, B. P. 1990, ApJ, 361, 309

O'Donoghue, D., Warner, B. \& Cropper, M. 1992, MNRAS, 258, 415

Oke, J. B., \& Gunn, J. E. 1983, ApJ, 266, 713

Robinson, E. L. 1979, in IAU Colloq. 53, White Dwarfs and Variable Degenerate Stars, ed. H. M. van Horn \& V. Weidemann (Rochester: Univ. Rochester), 343

Robinson, E. L., Kepler, S. O., \& Nather, R. E. 1982, ApJ, 259, 219

Standish, E. M. 1998, A\&A, 336, 381

Winget, D. E. 1981, Ph.D. thesis, Univ. Rochester 1998, J. Phys. Condensed Matter, 10, 11247

Winget, D. E., van Horn, H. M., Tassoul, M., Fontaine, G., Hansen, C. J., \& Carroll, B. W. 1982, ApJ, 252, L65

Wood, M. A. 1995, in Proc. 9th European Workshop, White Dwarfs, ed. D. Koester \& K. Werner (Berlin: Springer), 41

Wu, Y., \& Goldreich, P. 1999, ApJ, 519, 783

2001, ApJ, 546, 469 